\title{
Effect of Measurement of Total Organic Carbon Compounds to Improve Water Quality in the Shortest Time
}

\author{
Kalateh $\mathrm{M}^{*}$ and Barghamadi M \\ Iran-Khorasan Razavi-Mashhad - Rural Water and Wastewater Company - Central Laboratory, Iran
}

*Corresponding author: Kalateh M, Iran-Khorasan Razavi-Mashhad - Rural Water and Wastewater Company

- Central Laboratory, Iran, Tel: 0915508899, E-mail: kalatehm1@yahoo.com

Citation: Kalateh M, Barghamadi M (2018) Effect of Measurement of Total Organic Carbon Compounds to Improve Water Quality in the Shortest Time. J Public Health Hygiene Safety 1(1): 102. doi: 10.15744/2767-8792.1.102

Received Date: September 11, 2018 Accepted Date: December 17, 2019 Published Date: December 19, 2019

\section{Introduction}

One of the challenges and concerns of water industry experts and practitioners is to ensure that there is no bacterial contamination in water samples, especially drinking water samples. The second is to find out if there is a microbial biofilm layer in water resources such as wells, storage tanks, and even water distribution networks. The presence of biofilms in water resources and reservoirs reduces the quality of water. The study of internal walls of some resources, especially wells, is a bit difficult and costly. The solution to the knowledge of the formation of the lining layer or the lack of its maturity seemed indispensable. The heterotrophic bacteria in water, the main factor in the formation of the layer of biofilm layer, are in this type of source. On the other hand, the nature and structure of bacteria are carbon materials, so their presence can increase the amount of carbon in water. The total carbon contaminant is a parameter that can indirectly indicate the water quality used. In this study, an analysis of different sources of different water sources (different matrices), total organic carbon and heterotrophic bacteria were analyzed in the same sample and the relationship between these two parameters was determined. This study was carried out in different seasons of the year and in different geographical areas. As a result, by performing the whole organic carbon test, we were able to determine the formation or non-formation of biofilms in a particular source.

Keywords: Biofilm Layer; Total Organic Carbon; Heterotroph Bacteria

\section{Materials and Methods}

In this study, we tried to study the actual samples with different matrices to count the number of heterotrophic bacteria and the amount of organic carbon compounds. In fact, our goal was to examine whether it is possible to measure the amount of organic carbon and to know how small it can be, that the sample has a number of heterotrophic bacteria that can form the biofilm layer in the walls of reservoirs and wells and distribution pipes Water networks are coming.

To do this research we needed a specific schedule. Simultaneous prototypes were performed for bacteriological analysis (identification and counting of heterotrophic bacteria) and determination of microcrystalline carbon content in the same specimen [1]. Considering the fact that in the seasons and hot days of the year, the probability of bacterial growth and their activity was thought to be higher, during one year of our study, we selected different days from different seasons of the year for sampling. These days included different days of the week. The purpose of this kind of sampling program was to get the results from Generalize the analyzes to different days of the week, season and year with different temperature conditions [1]. We considered areas for sampling at a widespread level. In fact, samples were taken from different locations geographically and transported to the laboratory for less than 6 hours. Considering that the sources of supply and storage of drinking water are diverse; we have considered all the resources for doing research. In fact, samples were taken from wells, reservoirs of air and ground, and the distribution network. When removing the network, the blind spot network was considered and sampled.

Sample samples of carbon black microspheres were selected in dark colors [2]. Glasses the location of the laboratory in the furnace was placed at $400{ }^{\circ} \mathrm{C}$ to ensure that no carbon composite is present in the glass. After sampling, samples were taken in the laboratory. Two samples were taken on each sample. Bacterial analysis of counting of heterotrophic bacteria according to the book was carried out using Purple Plate method using Kant Agram Plate and incubation time of 48 hours. After incubation time, the number of colonies formed on the surface of the agar plate was counted using a counter-colony machine [2]. 
Another analysis was to determine the amount of organic carbon in the sample. To do this, the TOC Analyzer was used (Figure 1). After rapid sample transfer, the sample was injected to the device as soon as possible. The apparatus first analyzes the total amount of carbon and then the amount of inorganic carbon or inorganic carbon. The process of measuring the total carbon content of the device is that after the injection of the sample, the carbonaceous reactions in the sample are decomposed in a high-temperature furnace $\left(680^{\circ} \mathrm{C}\right)$ and converted to carbon dioxide. This gas is given by an infrared ray detector (NDIR) and displayed in peak form. The sub-peak level represents the concentration of carbon dioxide gas, which is in fact the total carbon content. To measure inorganic carbon, the sample is aerated with phosphoric acid and ZERO Air gas. The mineral compounds in the sample form as carbon dioxide gas and are detected by the detector. The difference between these two amounts of total carbon and inorganic carbon was determined by the amount of organic carbon that our target was investigating [3].

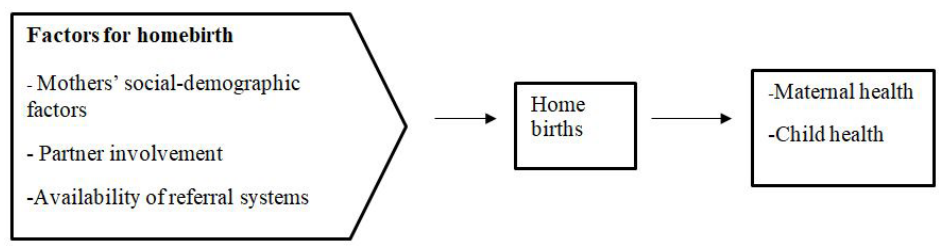

Figure 1: The process of converting carbon compounds in a sample of water into carbon dioxide gas and measuring it by Toc Analyzer

\section{Results}

In the research, the following results were obtained. The samples with high levels of contamination with heterotrophic bacteria also had high TOC levels. In fact, in the analyzed samples, there is a direct linear relationship between the two pareters. The higher the HPC contamination rate was, the higher the levels were with high carbon organic matter.

This was a new experience. We indirectly discovered the presence or absence of a biofilm layer in the water source, by identifying carbon dioxide in water samples from different sources of water. Measuring the amount of organic carbon in a sample, along with performing all the quality control activities of the test method and the device, with a three-time measurement of about 30 to 45 minutes, which is very helpful in reducing the amount of extraction.

\section{Discussion}

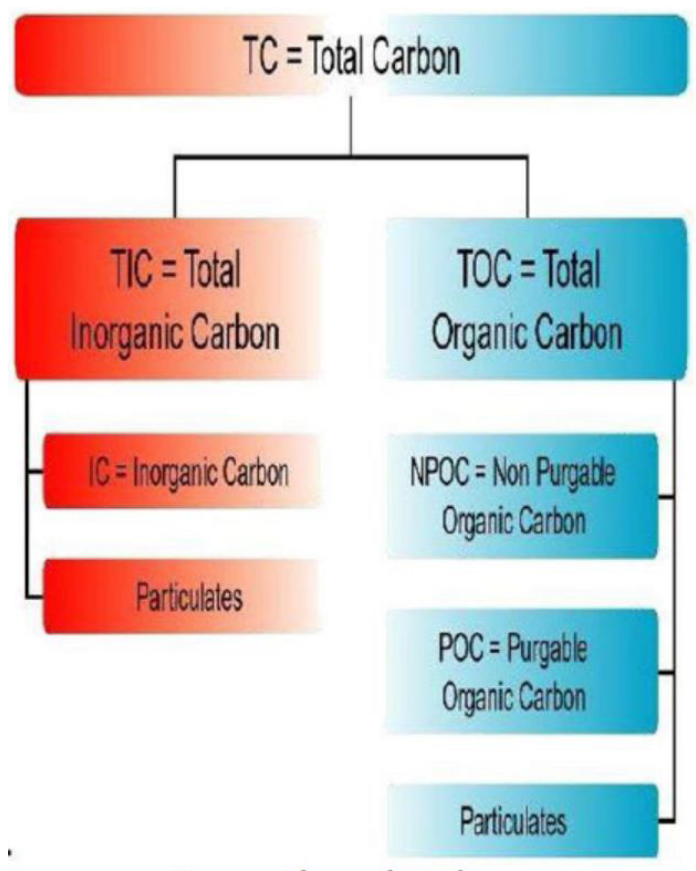

Figure 2: A linear relationship 
In studies conducted so far in the world, the relationship between total organic carbon values and parameters such as BOD and COD in a sewage sample has been considered, and much research has been done. These studies have shown that there is statistical relation between these values. And by applying a constant coefficient, these values can be obtained. We were able to move this connection to another. There was a significant relationship between the amount of heterotrophic bacteria and total organic carbon content and the formation of biofilm layer. A linear relationship (Figure 2).

\section{References}

1. Godec R (2000) The Performance Comparison of Ultrapure Water TOC Analyzers using an Automated Standard Addition Apparatus.

2. Total Organic Carbone Analyzer Manual (2014).

3. Robin S, Parnell, Roy-Keith Smith (22 $2^{\text {nd }}$ Edn) PART 5000, Aggregate Organic Constituents. Standard Methods for the Examination of Water and Waste Water.

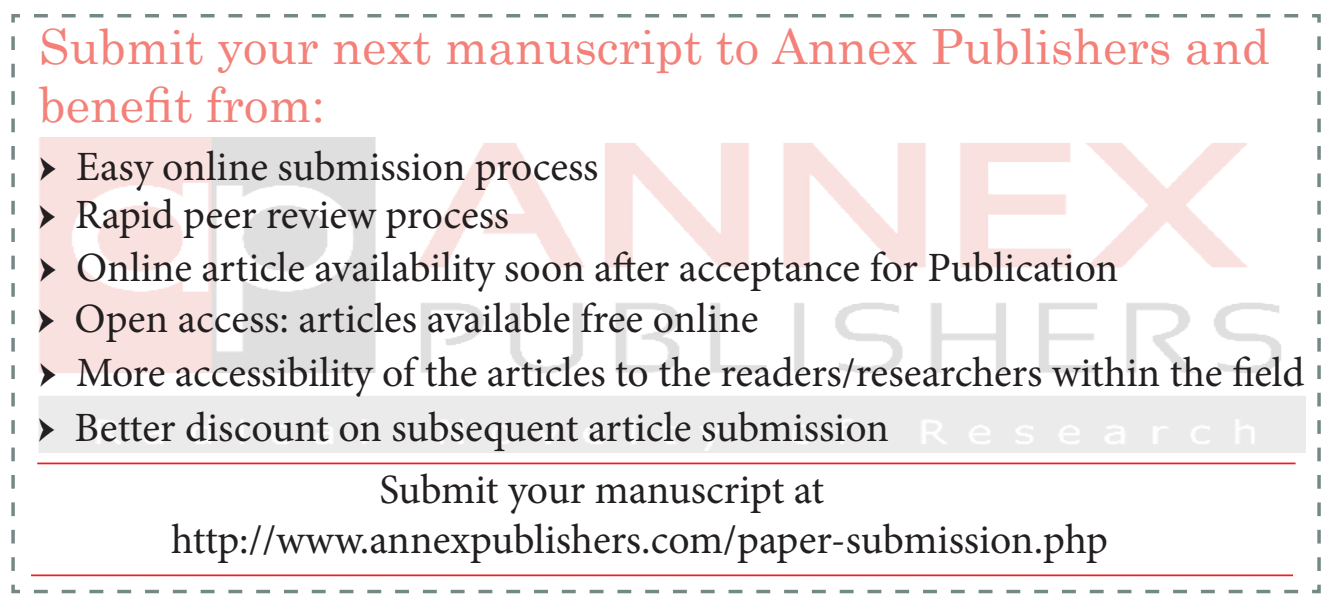

